\title{
Invoking Halanay inequality to conclude on closed-loop stability of a process with input-varying delay
}

\author{
D. Bresch-Pietri ${ }^{*}$ J. Chauvin ${ }^{* *}$ N. Petit ${ }^{*}$ \\ ${ }^{*}$ MINES ParisTech, Centre Automatique et Systèmes, Unité Mathématiques et \\ Systèmes, 60 Bd St-Michel, 75272 Paris, Cedex 06, France \\ (e-mail : delphine.bresch-pietri@mines-paristech.fr) \\ ** IFP Energies nouvelles, Département Contrôle, Signal et Système, 1 et 4 \\ avenue du Bois-Préau, 92852 Rueil-Malmaison, France
}

\begin{abstract}
At the light of a simple tutorial example, this paper discusses the merits of a recently introduced technique to control a class of systems with a delay depending on the past values of the control variables. The convergence proof of the proposed control strategy is obtained by a boundedness analysis of a class of delay differential equation, inspired by the Halanay inequality. The relations of the proposed technique with previous works from the literature on predictor-based controllers are discussed. The treated example is representative of a wide class of systems often observed in process control and distributed parameter systems.
\end{abstract}

\section{INTRODUCTION}

Numerous industrial applications involve a physical dead-time which reveals troublesome in the design and tuning of output feedback control laws, needed to achieve the still increasing expectations of dynamic performances. The source of this deadtime is that the sensors and the actuators are often not colocated. Such processes may involve transportation of material, such as gas in automotive engine (Bresch-Pietri et al. [2010]), liquid in mixing processes for chemical reactors (Harmand and Dochain [2005]) and heater collector plant (Sbarciog et al. [2008]), or blending (Chèbre et al. [2010]) and batch processes (Petit et al. [1998]). Very importantly, the lag directly depends on the manipulated variable. Therefore, the considered delay is inherently input-dependent and, then, non-linear.

A prime and very simple example of this type of non-linear dynamical systems is the shower system, or bath depicted in Fig. 1, for which a temperature regulation is considered ${ }^{1}$. In this example, the involved liquid holdups contained in the pipes are directly correlated to the history of the input flow rates. The holdup has a "group" velocity (due to the incompressible nature of the flow) which depends on the current value of the control. The temperature of the holdup is not spatially uniform, as it depends on the past values of the control which may not have been constant over a sufficiently long past. Due to its relative simplicity and its occurrence in everyday life, this example is often used to introduce time-delay systems in lectures and textbooks, like in Zhong [2006], and to illustrate some corresponding control challenges. Surprisingly, as similar problems involving input-varying delay, such as the crushingmill problem presented by Richard [2003], it seems that this problem has never been studied theoretically.

More generally, it seems that the stabilization problem of a nonlinear process, with input-dependent time-delay in the input, of

\footnotetext{
1 Of course, for this application, an open-loop result of asymptotic convergence can be straightforwardly obtained. Yet, closed-loop controllers lead to substantial transient improvements, which may be crucial in real-life applications.
}

type $\quad \dot{x}=f(x, u(t-D(u)))$

has never been theoretically considered. A classically proposed approach in such situations is to treat the delay dependence on the control by robustness, i.e. by neglecting this dependence as $D(u) \approx D(t)$ or even $D(u) \approx D$ and by requiring the controller to be able to deal with a certain level of model error.

Robust stability of systems with time-varying delay in the input has been widely studied lately : in Han and Gu [2001], He et al. [2007], Jiang and Han [2008] or Liu and Hu [2011], "memoryless" controllers are employed at the expense of substantial Linear Matrix Inequalities (LMIs) to check. On the other hand, predictor-based strategies have been proposed to improve closed-loop dynamic performance,(see Smith [1959], Artstein [1982], Manitius and Olbrot [2002]). Such techniques, which are widely used for a constant input time-delay (see for instance Jankovic [2008], Krstic [2008b], Mondié and Michiels [2003], Moon et al. [2001] or Gu and Niculescu [2003] and Richard [2003] and the reference therein) are less popular for time-varying ones. Yet, in Nihtila [1991] or, more recently, in Krstic [2009], a time-varying delay version of predictor-based control has been proposed. To compensate the input delay, this prediction is calculated over a time window of which length matches the value of the future delay ${ }^{2}$. In other words, it is required to be able to predict the future variations of the delay, which is not always practically achievable when the delay depends on the input.

For this reason, in this paper, although we use a prediction technique, we do not aim at exactly compensating the delay. We blend the previously described techniques and propose a robust compensation approach as has been done recently in Yue and Han [2005] for example. We follow the overture proposed in Krstic [2008a] and Krstic [2009] to analyze the stability of linear input time-delay systems (and which have been devel-

\footnotetext{
2 In details, defining the delay operator $\phi(t)=t-D(t)$ and assuming that its inverse is well-defined, exact delay-compensation is obtained with the feedback law $U(t)=K X_{\text {pred }}\left(\phi^{-1}(t)\right)$.
} 
oped in Bresch-Pietri et al. [To appear] and in Bresch-Pietri and Krstic [2009] to address uncertainties to constant input time-delays in various contexts). Here, we extend these tools to linear systems with time-varying delay in the input. To address the particular problem under consideration, we explicitly relate the obtained result with the regulation of the considered class of input-varying delay processes. Technically, we guarantee the boundedness of the delay variations by relating it to the boundedness of the control tracking error, over successive time windows, which is in turn achieved using a modified Halanay inequality. To the best of the authors knowledge, this relation between prediction-based control law and input-dependency of the delay has never been studied. This is the main contribution of the paper.

The paper is organized as follows. In Section 2, we briefly describe the mixing process under consideration, before designing in Section 3 a prediction-based controller using a tailored change of time scale. After having presented this controller, we focus on Section 4 on its conditions of realization invoking Halanay boundedness results. Finally, a proof of convergence of the proposed controller for linear time-varying delay systems is provided in Appendix.

\section{PROBLEM STATEMENT}

Consider the bath system represented in Fig. 1, where the average bath temperature $T_{f}$ is the result of a mixing between a cold water source (flow rate $u_{1}$ and temperature $T_{1}$ ) and a warm one $\left(u_{2}\right.$ and $\left.T_{2}\right)$. For comfort purposes, the user of the bath wishes to obtain a desired temperature $T_{r e f}$, preferably without over- or under-shoots, and as fast as possible (namely, close to the minimum reaching time introduced by the transport delay through the pipe).

For sake of simplicity, we assume here that the change of the faucet position is instantaneous and that the bath volume $V$ is constant. Further, we assume that the position of the cold water faucet is fixed. Without loss of generality, $u_{1}=1, T_{1}=0, T_{2}=1$, $T_{\text {ref }} \in\left[0,1\left[, V=1\right.\right.$ and we denote $u=u_{2} \in[0,1]$. Assuming that the position of the warm water faucet is directly correlated to the flow rate through static relations, $u$ is then considered as the input variable. Finally, we assume that the bath temperature $T_{f}$ is available for measurement.

\subsection{Balance equations}

Assuming that the mixing at the node is instantaneous, the punctual temperature at this node can simply be expressed as

$$
T_{m o y}(t)=\frac{u(t)}{1+u(t)}
$$

We neglect the heat losses during the flow transport from the node to the pipe output, namely

$$
T_{\text {out }}(t)=T_{\text {moy }}(t-D(t))
$$

where $D(t)$ accounts for the varying transport delay implicitly defined by

$$
V_{P}=\int_{t-D(t)}^{t}(1+u(s)) d s
$$

with $V_{P}$ the pipe volume. Further, a heat balance yields, using (2) and the fact that the bath volume $V=1$ is constant,

$$
\frac{d}{d t}\left(T_{f}\right)=(1+u(t))\left[-T_{f}(t)+T_{m o y}(t-D(t))\right]
$$

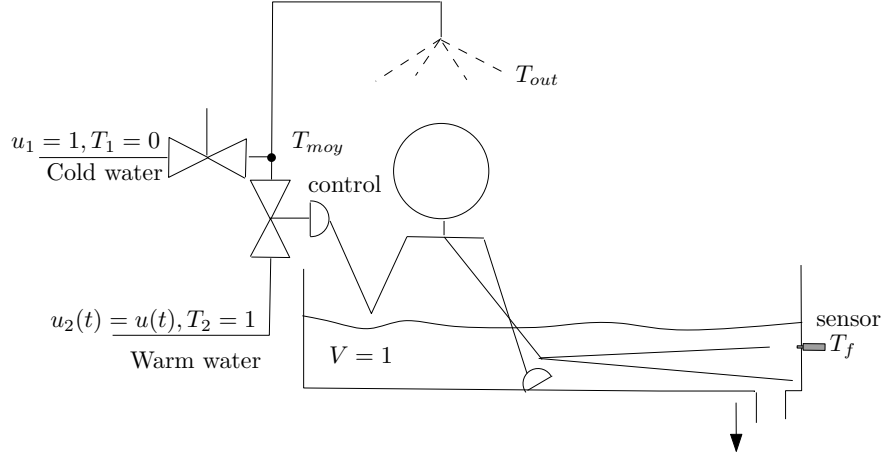

Fig. 1. The studied shower system.

\subsection{Control objective}

The control objective is to have system (4) track the given temperature set-point $T_{\text {ref }}$ as fast as possible, taking into account the above constraints. To reach this goal, we develop a prediction-based control law taking advantage of the knowledge of the implicit delay variation law (3).

\section{CONTROL DESIGN}

\subsection{Alternative system representation}

To design an explicit prediction-based feedback law, we first introduce the following change of time

$$
\tau \stackrel{\text { def }}{=} h(t)=\frac{1}{1+\bar{u}} \int_{0}^{t}(1+u(s)) d s+\tau_{0}
$$

where $\bar{u}$ is a normalization factor chosen as $\bar{u}=\frac{T_{\text {ref }}}{1-T_{\text {ref }}}$ and where $\tau_{0} \geq 0$ is a given constant. This leads to the alternative linear system

$$
\frac{d X}{d \tau}(\tau)=(1+\bar{u})\left[-X(\tau)+T_{\text {moy }}\left(\tau-D_{2}(\tau)\right)\right]
$$

where the alternative system state is defined as $X(\tau)=T_{f}(t)$ and a new delay has been introduced as

$$
D_{2}(\tau)=\tau-t+D(t)=\tau-h^{-1}(\tau)+D\left(h^{-1}(\tau)\right)
$$

In this new time scale ${ }^{3},(6)$ is a linear input-delay system with constant parameters. Rewriting the alternative delay as $D_{2}(h(t))=h(t)-t+D(t)$ and considering both (3) and (5), one can observe that this expression is still input-dependent, but in a much more complex way. The well-posedness of (5)-(7) is proved in Bresch-Pietri et al. [2012].

\subsection{Control design for the alternative system (6)}

We now formulate the following theorem, a proof of which is provided in Appendix.

Theorem 1. Consider the closed-loop system

$$
\begin{aligned}
& \dot{X}(t)=A X(t)+B U(t-D(t)) \\
& U(t)=U^{r}-K X^{r}+K\left[e^{A D(t)} X(t)+\int_{t-D(t)}^{t} e^{A(t-s)} B U(s) d s\right]
\end{aligned}
$$

where $X \in \mathbb{R}^{n}, U$ is scalar, the pair $(A, B)$ is controllable, $K$ is chosen such that $A+B K$ is Hurwitz, $\left(X^{r}, U^{r}\right)$ is a given equi-

\footnotetext{
3 A different constant scale factor could be introduced in (5) to simplify the expression of (6) at the expense of later complexity in the analysis of closedloop behavior.
} 
librium set-point and $D: \mathbb{R}_{+} \rightarrow[\underline{D}, \bar{D}]$ is a time-differentiable function. Then, there exists $\left.\delta^{*} \in\right] 0 ; 1[$ such that, provided

$$
\forall t \geq 0, \quad|\dot{D}(t)|<\delta^{*},
$$

the plant (8a) exponentially converges to the equilibrium $X^{r}$.

Before studying the application of this theorem to our "shower", we wish to comment it. Control law (8b) is a predictor directly inspired by the constant delay case, forecasting values of the state over a time window of varying length $D(t)$. Of course, exact compensation of the delay is not achieved with this controller ${ }^{4}$ and equation (9) can be interpreted as a condition for robust compensation achievement. Namely, if the delay varies sufficiently slowly, its current value $D(t)$ used for prediction can be expected to be close enough to its future values, and the corresponding prediction will be accurate enough to ensure the stabilization of the plant.

Interestingly, the exact same condition is stated in Yue and Han [2005], where the delay is also assumed to be timedifferentiable. Yet, the approach designed in Yue and Han [2005] differs from ours in the way that a constant average delay value is used for control ${ }^{5}$, which should naturally result into performance losses.

\subsection{Control law for the original plant}

Theorem 2. Consider the closed-loop system consisting of the plant (4), with the input-delay defined through (1)-(3), and the control law

$$
\begin{aligned}
u(t)= & S a t_{[0,1]}\left\{\frac{T_{\text {moy }}(t)}{1-T_{\text {moy }}(t)}\right\} \\
T_{\text {moy }}(t)= & (1+k) T_{\text {ref }}-k\left[e^{-(1+\bar{u}) D_{2}(t)} T_{f}\left(h^{-1}(t)\right)\right. \\
& \left.+(1+\bar{u}) \int_{t-D_{2}(t)}^{t} e^{-(1+\bar{u})(t-s)} T_{\text {moy }}(s) d s\right] \\
D_{2}(t)= & t-h^{-1}(t)+D\left(h^{-1}(t)\right)
\end{aligned}
$$

where the function $h$ has been defined in (5) and $S a t_{[0,1]}$ represents the saturation operator. There exists $k^{*} \in \mathbb{R}_{+}^{*}$, potentially depending on the initial condition and the input past on a time window of finite length $u_{0}$, such that, for $k \in\left[0, k^{*}\right.$,

$$
T_{f}(t) \underset{t \rightarrow \infty}{\longrightarrow} T_{r e f}
$$

This result is not surprising : Theorem 1 requires the delay to vary sufficiently slowly, while, on the other hand, the delay variations implicitly depend on the control input, variations which aggressiveness is scaled by the gain $k$. Then, restricting the input variations by choosing the feedback gain sufficiently small seems like a natural solution. Further, as the "shower" under consideration is stable, it is possible to choose $k$ as small as desired and in particular to ensure (9).

An important point for implementability is the causality of the control law (10), as the inverse transformation $h^{-1}$ is employed. This point can still be guaranteed (see Bresch-Pietri et al.

\footnotetext{
4 To do so, one would need to consider a time window of which length would match exactly the value of the future delay, as it is made in Krstic [2009]. In details, defining the delay operator $\phi(t)=t-D(t)$ and assuming that its inverse exists and is available, exact delay-compensation is obtained with the feedback law $U(t)=K X\left(\phi^{-1}(t)\right)$. Yet, this requires to be able to predict the future variation of the delay via $\phi^{-1}(t)$, which is not practically achievable for an input-varying delay.

5 More precisely, the control law is designed after a reduction model using this average value.
}

[2012] for details). For strict implementability, an expression of the bound $\delta^{*}$ is provided in Appendix. Nevertheless, as this formulation is obtained based on a Lyapunov analysis, it turns out to be quite conservative and brings little help in practice.

The interested reader may refer to the companion paper BreschPietri et al. [2012] for simulation results highlighting these points, and emphasizes the interest of this control law by comparison with a simple proportional controller.

We now detail the proof of Theorem 2 .

\section{PROOF OF THEOREM 2 - HALANAY INEQUALITY}

As the alternative plant (6) directly matches the framework of Theorem 1 and as the prediction-based control law (11) is a direct application of ( $8 \mathrm{~b})$ to the considered case, the condition (9) is sufficient to obtain the desired convergence. Indeed, if this condition is fulfilled, applying Theorem 1 , one can ensure that the system state in (6) asymptotically converges to $T_{r e f}$. As the diffeomorphism $h$ is unbounded as its argument goes to infinity, this also involves the asymptotic convergence of $T_{f}$. Here, this condition can be written as

$$
\left|\frac{\partial D_{2}}{\partial \tau}(\tau)\right|<\delta^{*}
$$

In this section, we focus on a general manner to guarantee this condition ${ }^{6}$. Some misleading notations are given in Appendix.

\subsection{Reformulation of (13)}

First, from (7) and (5), one can easily obtain an analytic expression of this partial derivative

$$
\frac{\partial D_{2}}{\partial \tau}(\tau)=\frac{\partial D_{2}}{\partial \tau}(h(t))=1-[1-\dot{D}(t)] \frac{1+\bar{u}}{1+u(t)}
$$

Further, taking a time-derivative of the implicit equation (3), one gets,

$$
1-\dot{D}(t)=\frac{1+u(t)}{1+u(t-D(t))}
$$

and then, substituting,

$$
\begin{aligned}
\left|\frac{\partial D_{2}}{\partial \tau}(\tau)\right|<\delta^{*} & \Leftrightarrow\left|1-\frac{1+\bar{u}}{1+u(t-D(t))}\right|<\delta^{*} \\
& \Leftrightarrow \frac{1+\bar{u}}{1+\delta^{*}}<1+u(t-D(t))<\frac{1+\bar{u}}{1-\delta^{*}} \\
& \Leftrightarrow \frac{1-\delta^{*}}{1+\bar{u}}<1-T_{\text {moy }}(t-D(t))<\frac{1+\delta^{*}}{1+\bar{u}}
\end{aligned}
$$

Finally, exploiting the equilibrium relation between $\bar{u}$ and $T_{r e f}$, this condition can be rewritten as the inequality bearing on the input

$$
\left|T_{\text {moy }}(t-D(t))-T_{r e f}\right|<\delta^{*} \frac{1}{1+\bar{u}}=\delta^{*}\left(1-T_{r e f}\right)
$$

As a result, the inequality (13) bearing on the delay timederivative is equivalent to the previous one on the input, which is consistent with the input-dependency on the delay. To study this last inequality, we now introduce some intermediate results.

\subsection{Halanay inequality and related boundedness results}

We first recall the following result (Halanay [1966], Ivanov et al. [2002]) ${ }^{7}$.

\footnotetext{
6 which has been guaranteed in Bresch-Pietri et al. [2012] accounting for a particularity of this system which is to presend only bounded variables (flows mainly).

7 More precisely, in Halanay [1966], this result is stated for $a>b>0$.
} 
Lemma 1. (Halanay inequality) Consider a positive continuous real-value function $x$ such that, for some $t_{0} \in \mathbb{R}$,

$$
\dot{x}(t) \leq-a x(t)+b \max x_{t}, \quad t \geq t_{0}
$$

with $a \geq b \geq 0$ and where the notation $x_{t}: s \in[-\bar{D}, 0] \mapsto x(t+s)$ has been used. Then there exists $\gamma \geq 0$ such that

$$
\forall t \geq t_{0}, \quad x(t) \leq \max x_{t_{0}} e^{-\gamma\left(t-t_{0}\right)}
$$

A direct application of this result is the lemma below.

Lemma 2. Consider a delay differential equation of the form

$$
\left\{\begin{array}{c}
\dot{x}(t)+a x(t)+b h\left(t, x_{t}\right)=0, \quad t \geq t_{0} \\
x_{t_{0}}=\phi \in \mathscr{C}^{0}([-\bar{D}, 0])
\end{array}\right.
$$

where the continuous functional $h$ satisfies the sup-norm relation

$$
\left|h\left(t, x_{t}\right)\right| \leq \max \left|x_{t}\right|
$$

Then if $a \geq b \geq 0$, there exists $\gamma \geq 0(\gamma=0$ if $a=b)$ such that every solution of (15) satisfies

$$
\forall t \geq t_{0}, \quad|x(t)| \leq \max \left|x_{t_{0}}\right| e^{-\gamma\left(t-t_{0}\right)}
$$

Proof Consider $x$ a non-trivial continuous solution ${ }^{8}$ of (15) which does satisfy the inequality

$$
\frac{d|x(t)|}{d t}+a|x(t)| \leq b \max \left|x_{t}\right| \quad \text { provided }|x(t)| \neq 0
$$

Following the own proof of Halanay [1966], define $y=k e^{-\gamma\left(t-t_{0}\right)}$ with $k>0$ and $\gamma$ chosen such that $y$ satisfies the corresponding differential equation $^{9}$

$$
\dot{y}(t)=-a y(t)+b \max y_{t}, \quad t \geq t_{0} \quad \text { and } \quad y_{t_{0}}=k
$$

Now, we define the difference $z=y-|x(t)|$ which is a continuous function and we are interested in its sign change. We choose $k>\max \left|x_{t_{0}}\right|$ to ensure that $z\left(t_{0}\right)>0$ for $t \in\left[t_{0}-\bar{D}, t_{0}\right]$. The function $z$ being continuous, we define

$$
t_{1}=\inf \left\{t>t_{0} \mid z(t)=0\right\} \in \mathbb{R} \cup\{\infty\}
$$

Assume that $t_{1}<\infty$. Then $\left|x\left(t_{1}\right)\right|=y\left(t_{1}\right)>0$ by the analytic expression of $y$ and, by continuity, there exists an open set ]$a_{1}, b_{1}\left[\right.$ such that $\left.t_{1} \in\right] a_{1}, b_{1}[$ and $|x(t)|>0$ for $t \in] a_{1}, b_{1}[$. Consequently, $z$ is continuously differentiable on $] a_{1}, b_{1}[$ and satisfies

$$
\forall t \in] a_{1}, b_{1}\left[, \quad \dot{z}(t)+a z(t) \geq b\left(\max y_{t}-\max \left|x_{t}\right|\right)\right.
$$

Then, $\dot{z}\left(t_{1}\right) \geq \max y_{t_{1}}-\max \left|x_{t_{1}}\right|>0$, by definition of $t_{1}$. Yet, one has

$$
\dot{z}\left(t_{1}\right)=\lim _{t \rightarrow t_{1}^{-}} \frac{z(t)-z\left(t_{1}\right)}{t-t_{1}}=\lim _{t \rightarrow t_{1}^{-}} \frac{z(t)}{t-t_{1}} \leq 0 \text { as } z(t) \geq 0 \text { on }\left[t_{0}, t_{1}\right]
$$

We finally conclude that $t_{1}=\infty$. Then, $\forall t \geq t_{0}, z(t)>0$ and

$$
\forall \varepsilon>0 \quad \forall t \geq t_{0} \quad|x(t)|<\left(\max \left|x_{t_{0}}\right|+\varepsilon\right) e^{-\gamma\left(t-t_{0}\right)}
$$

which gives the result.

To match with the considered framework, we now state a local version of the previous lemma.

Corollary 1. Consider a delay differential equation of the form

$$
\left\{\begin{aligned}
\dot{x}(t) & +a x(t)+b h\left(t, x_{t}\right)=0, \quad t \geq t_{0} \\
x_{t_{0}} & =\phi \in \mathscr{C}^{0}([-\bar{D}, 0])
\end{aligned}\right.
$$

where $h$ is a continuous functional satisfying, on an open neighborhood $V$ of the origin, the sup-norm relation

$$
\forall x_{t}:[-\bar{D}, 0] \mapsto V, \quad\left|h\left(t, x_{t}\right)\right| \leq \max \left|x_{t}\right|
$$

\footnotetext{
8 The continuity (and even more) is obtained by assuming $\phi$ smooth enough.

${ }^{9} \gamma \geq 0$ is the unique solution on $\left[0, \infty\left[\right.\right.$ of $a-\gamma=b e^{\gamma \bar{D}}$.
}

Then, if the initial value $\phi$ maps $[-\bar{D}, 0]$ to $V$ and if $a \geq b \geq 0$, then there exists $\gamma \geq 0(\gamma=0$ if $a=b)$ such that every solution satisfies

$$
\forall t \geq t_{0}, \quad|x(t)| \leq \max \left|x_{t_{0}}\right| e^{-\gamma\left(t-t_{0}\right)}
$$

Proof The essence of the proof is similar to the one of Lemma 2. Consider again a non trivial solution $x$ such that $x_{t_{0}}:[-\bar{D}, 0] \mapsto V, y(t)=k e^{-\gamma\left(t-t_{0}\right)}$ s. t. $\dot{y}(t)=-a y(t)+b \max y_{t}$ for $t \geq t_{0}$ and $z=y-|x|$ with $z\left(t_{0}\right)>0$. Define again $t_{1}=\inf \left\{t>t_{0} \mid z(t)=0\right\} \in \mathbb{R} \cup\{\infty\}$. If $k>\max \left|x_{t_{0}}\right|$ and $k \in V$ ( $k$ always exists as $V$ is an open set by assumption), one can ensure that $x(t) \in V$ for $t \in\left[t_{0}, t_{1}\right]$ from the fact that $y(t) \in V$, for $t \in\left[t_{0}, t_{1}\right]$. Then, $\forall t \geq t_{0} \quad z(t)>0$ and the result directly follows.

4.3 Application to establish the boundedness of input-delay systems with the prediction-based control law

Lemma 3. Consider a continuous real-valued $f$ and a differentiable real-valued function $\psi$ such that

$$
\begin{aligned}
f(t) & =-k\left[e^{-\alpha D(t)} \psi(t)+\alpha \int_{t-D(t)}^{t} e^{-\alpha(t-s)} f(s) d s\right] \\
\dot{\psi}(t) & =\alpha[-\psi(t)+f(t-D(t))]
\end{aligned}
$$

where $k>0$ and $\alpha>0$ are constant and $D:[0, \infty[\rightarrow[\underline{D}, \bar{D}](0<$ $\underline{D}<\bar{D})$ is a time-differentiable function such that

$$
\forall t \geq 0, \quad|\dot{D}(t)| \leq \beta \max _{s \in[t-\bar{D}, t]}|f(s)|
$$

If there exists $t_{0} \in \mathbb{R}$ s. t. $\forall t \in\left[t_{0}-\bar{D}, t_{0}\right]|f(t)|<M / \beta$ with $M<1$ and $\beta>0$, then

$$
\forall t \geq t_{0}, \quad|f(t)|<M / \beta
$$

Proof Taking the time-derivative of (20) and using relation (21), one can obtain that $f$ satisfies the following delay differential equation

$$
\begin{aligned}
\dot{f}(t)+\alpha(1+k) f(t)= & -\alpha \dot{D}(t)\left(f(t)+k e^{-\alpha D(t)} f(t-D(t))\right. \\
& \left.+k \alpha \int_{t-D(t)}^{t} e^{-\alpha(t-s)} f(s) d s\right)
\end{aligned}
$$

Then, defining $a=b=\alpha(1+k), V=]-M / \beta, M / \beta[$ and

$$
\begin{aligned}
& h\left(t, x_{t}\right)=\frac{\dot{D}(t)}{1+k} \times \\
& {\left[f(t)+k\left(e^{-\alpha D(t)} f(t-D(t))+\alpha \int_{t-D(t)}^{t} e^{-\alpha(t-s)} f(s) d s\right)\right]}
\end{aligned}
$$

one can apply Corollary 1 as, using (22),

$$
\begin{aligned}
|h(x, t)| & \leq\left|\frac{\beta \max \left|f_{t}\right|}{1+k}\left(1+k\left[e^{-\alpha D(t)}+1-e^{\alpha D(t)}\right]\right) \max \right| f_{t} \mid \\
& \leq \beta \max \left|f_{t}\right|^{2} \leq \max \left|f_{t}\right| \text { for } f_{t}:[-\bar{D}, 0] \mapsto V
\end{aligned}
$$

and conclude that, $\forall t \geq t_{0}, f(t) \in V$, i.e. $\forall t \geq t_{0}, f(t)<\frac{M}{\beta}$.

\subsection{Proof of Theorem 2}

Observing (10), we aim at using Lemma 3 with $\alpha=1+\bar{u}$, $\psi(t)=T_{f}\left(h^{-1}(t)\right)-T_{r e f}$ and $f=T_{m o y}-T_{\text {ref }}$. Indeed, taking a time-derivative of (5) evaluated at time $h^{-1}(t)$, one obtains $\frac{d h^{-1}(t)}{d t}=\frac{1+\bar{u}}{1+u\left(h^{-1}(t)\right)}$ and one can realize that $\psi$ satisfies

$$
\dot{\psi}(t)=\frac{d T_{f}}{d t}\left(h^{-1}(t)\right) \frac{d h^{-1}(t)}{d t}
$$




$$
\begin{aligned}
& =\left(1+u\left(h^{-1}(t)\right)\right)\left[-\psi(t)+f\left(h^{-1}(t)-D\left(h^{-1}(t)\right)\right)\right] \frac{d h^{-1}(t)}{d t} \\
& =(1+\bar{u})\left[-\psi(t)+f\left(t-D_{2}(t)\right)\right]
\end{aligned}
$$

Consequently, now we just need to study the alternative delay $D_{2}(t)$. Taking a time-derivative of (7) and of the implicit relation (3), both evaluated at time $h^{-1}(t)$, one gets

$$
\begin{aligned}
\dot{D_{2}}(t) & =1-\frac{d}{d t}\left[h^{-1}(t)-D\left(h^{-1}(t)\right)\right] \\
& =1-\frac{1+\bar{u}}{1+u\left(h^{-1}(t)-D\left(h^{-1}(t)\right)\right)} \\
& =\frac{T_{\text {moy }}\left(t-D_{2}(t)\right)-T_{r e f}}{1-T_{\text {ref }}}=\frac{f\left(t-D_{2}(t)\right)}{1-T_{\text {ref }}}
\end{aligned}
$$

Therefore, $\left|\dot{D}_{2}(t)\right| \leq \beta \max \left|f_{t}\right|$ with $\beta=\frac{1}{1-T_{\text {ref }}}>0$ and $f_{t}$ defined on $\left[-\bar{D}_{2}(t), 0\right]$. Further, the previous results can be easily extended to the case where the delay upper bound is time-varying and one can observe that $\delta^{*}<1$. Then, one deduces from Lemma 3 that, for $t \geq t_{0},|f(t)|<\delta^{*} / \beta$ provided $\left|f\left(t_{0}+s\right)\right|<\delta^{*} / \beta, s \in\left[-\bar{D}_{2}\left(t_{0}\right), 0\right]$ (with $\bar{D}_{2}\left(t_{0}\right)$ denoting the upper bound at time $\left.t_{0}\right)$. Then, (14) is equivalent to the less requiring condition

$$
\max _{s \in\left[-\overline{D_{2}}(0), 0\right]}\left|T_{\text {moy }}(t)-T_{\text {ref }}\right|<\delta^{*}\left(1-T_{\text {ref }}\right)
$$

with $\overline{D_{2}}(0)=\bar{D}+\tau_{0}$. Finally, from (11), this condition is fulfilled provided $0 \leq k<k^{\star}$ with $k^{\star}>0$.

\section{CONCLUSION}

This paper proposes a prediction-based control strategy for a system with an input-dependent delay. This strategy uses a proposed result on robust prediction-based control of time-varying delay systems (Theorem 1) and relates it to a small-gain condition (Theorem 2) to account for the delay dependence on the input variable, through the considered integral equation modeling the transport phenomenon. This relation is established by some extensions of Halanay inequality formulating boundedness properties of a class of delay differential equations.

The resulting controller can be reasonably interpreted as a promising way to improve performance of a large amount of industrial processes involving input-dependent delay.

\section{Appendix A. NOTATIONS}

- $\mathscr{C}^{0}(S)$ denotes the set of continuous functions on the set $S$

- $x_{t}$ refers to the function $x_{t}: s \in[-\bar{D}, 0] \rightarrow x(t+s)$ for a given function $x$

\section{Appendix B. PROOF OF THEOREM 1}

In the following, we use the Lyapunov tools introduced in Krstic [2008a] to analyze the stability of input time-delay systems and which are based on a backstepping transformation of a certain actuator state defined for constant delays ${ }^{10}$. First, to extend them to the time-varying delay case, we introduce the distributed input $u(x, t)=U(t+D(t)(x-1)), x \in[0,1]$, which enables to rewrite plant (8a) as

$$
\left\{\begin{aligned}
\dot{X}(t) & =A X(t)+B u(0, t) \\
D(t) u_{t}(x, t) & =u_{x}(x, t)+\dot{D}(t)(x-1) u_{x}(x, t) \\
u(1, t) & =U(t)
\end{aligned}\right.
$$

\footnotetext{
10 This transformation is made to convert the plant $\dot{X}(t)=A X(t)+B u(0, t)$, $D u_{t}(x, t)=u_{x}(x, t)$ with the boundary condition $u(1, t)=U(t)$ into the target system $\dot{X}(t)=(A+B K) X(t)+B w(0, t), D w_{t}(x, t)=w_{x}(x, t)$ with the boundary condition $w(1, t)=0$.
}

In details, the input delay is now represented as a coupling with a transport partial differential equation (PDE) driven by the input and where the convection speed varies both with space and time.

Pursuing the mentioned approach, we now define the following transformed distributed input, based on the tracking error $\tilde{X}(t)=X(t)-X^{r}$ and the distributed input error $e(x, t)=$ $u(x, t)-U^{r}$,

$w(x, t)=e(x, t)-D(t) K \int_{0}^{x} e^{A D(t)(x-y)} B e(y, t) d y-K e^{A D(t) x} \tilde{X}(t)$

This Volterra integral equation of the second kind is designed to fulfill $w(1, t)=0$, compliantly with the control choice formulated earlier. The error plant corresponding to (8a)-(8b) can then be expressed as

$$
\left\{\begin{aligned}
\dot{\tilde{X}}(t) & =(A+B K) \tilde{X}(t)+B w(0, t) \\
D(t) w_{t}(x, t) & =w_{x}(x, t)-\dot{D}(t) D(t) g(x, t) \\
w(1, t) & =0
\end{aligned}\right.
$$

where the function $g$ is defined as

$$
\begin{aligned}
g(x, t)= & \frac{(1-x)}{D(t)} e_{x}(x, t)+K A x e^{A D(t) x} \tilde{X}(t) \\
& +K \int_{0}^{x} e^{A D(t)(x-y)}(I+A D(t)(x-y)) B e(y, t) d y \\
& +K \int_{0}^{x} e^{A D(t)(x-y)} B(y-1) e_{x}(y, t) d y
\end{aligned}
$$

For the Lyapunov analysis below, we also need the governing equation of the spatial derivative of the transformed distributed input $w_{x}$

$$
\begin{aligned}
D(t) w_{x t}(x, t) & =w_{x x}(x, t)-\dot{D}(t) D(t) g_{x}(x, t) \\
w_{x}(1, t) & =\dot{D}(t) D(t) g(1, t)
\end{aligned}
$$

We can now start the Lyapunov analysis and introduce the following Lyapunov-Krasovski functional

$$
\begin{aligned}
V(t)= & \tilde{X}(t)^{T} P \tilde{X}(t)+b_{1} D(t) \int_{0}^{1}(1+x) w(x, t)^{2} d x \\
& +b_{2} D(t) \int_{0}^{1}(1+x) w_{x}(x, t)^{2} d x
\end{aligned}
$$

where the symmetric matrix $P$ satisfies the following Lyapunov equation $P(A+B K)+(A+B K)^{T} P=-Q$ for a given symmetric definite positive matrix $Q$ (we denote $\lambda_{\text {min }}(Q)$ its minimum eigenvalue). Taking a time-derivative of $V$, we get after some integrations by parts and bounding

$$
\begin{aligned}
& \dot{V}(t)=-\tilde{X}(t)^{T} Q \tilde{X}(t)+2 \tilde{X}(t)^{T} P B w(0, t)+b_{1}\left(-w(0, t)^{2}\right. \\
& \left.-\|w(t)\|^{2}\right)+b_{2}\left(2 w_{x}(1, t)^{2}-w_{x}(0, t)^{2}-\left\|\hat{w}_{x}(t)\right\|^{2}\right) \\
& +2 b_{1}|\dot{D}(t)|\left|D(t) \int_{0}^{1}(1+x) w(x, t) g(x, t) d x\right| \\
& +2 b_{2}|\dot{D}(t)|\left|D(t) \int_{0}^{1}(1+x) w_{x}(x, t) g_{x}(x, t) d x\right| \\
& +|\dot{D}(t)|\left(b_{1} \int_{0}^{1}(1+x) w(x, t)^{2} d x+b_{2} \int_{0}^{1}(1+x) w_{x}(x, t)^{2} d x\right)
\end{aligned}
$$

To bound the remaining positive terms, one can introduce the inverse transformation of (B.1)

$$
\begin{aligned}
e(x, t)= & w(x, t)+D(t) K \int_{0}^{x} e^{(A+B K) D(t)(x-y)} B w(y, t) d y \\
& +K e^{(A+B K) D(t) x} \tilde{X}(t)
\end{aligned}
$$


and its spatial derivative to obtain the following inequalities, using Young's and Cauchy-Schwartz's inequalities,

$$
\begin{aligned}
& 2\left|D(t) \int_{0}^{1}(1+x) w(x, t) g(x, t)\right| d x \\
& \leq M_{1}\left(|\tilde{X}(t)|^{2}+\|w(t)\|^{2}+\left\|w_{x}(t)\right\|^{2}\right) \\
& 2 w_{x}(1, t)^{2} \leq M_{2}|\dot{D}(t)|^{2}\left(|\tilde{X}(t)|^{2}+\|w(t)\|^{2}+\left\|w_{x}(t)\right\|^{2}\right) \\
& 2\left|D(t) \int_{0}^{1}(1+x) w_{x}(x, t) g_{x}(x, t)\right| d x \\
& \leq M_{3}\left(|\tilde{X}(t)|^{2}+\|w(t)\|^{2}+\left\|w_{x}(t)\right\|^{2}+w_{x}(0, t)^{2}\right)
\end{aligned}
$$

where $M_{1}, M_{2}$ and $M_{3}$ are positive constant, the expression of which is not provided here. Using Young's inequality and the previous ones and defining $V_{0}(t)=|\tilde{X}(t)|^{2}+\|w(t)\|^{2}+$ $\left\|w_{x}(t)\right\|^{2}$, it is straightforward to get

$$
\begin{aligned}
& \dot{V}(t) \leq-\frac{\lambda_{\min }(Q)}{2}|\tilde{X}(t)|^{2}-\left(b_{1}-\frac{2|P B|^{2}}{\lambda_{\min }(Q)}\right) w(0, t)^{2} \\
& -b_{1}\|w(t)\|^{2}-b_{2}\left\|w_{x}(t)\right\|^{2}-b_{2}\left(1-M_{3}|\dot{D}(t)|\right) w_{x}(0, t)^{2} \\
& +|\dot{D}(t)|\left(b_{1} M_{1}+b_{2} M_{2}|\dot{D}(t)|+b_{2} M_{3}+2 b_{1}+2 b_{2}\right) V_{0}(t)
\end{aligned}
$$

Consequently, choosing $b_{1} \geq 2|P B|^{2} / \lambda_{\min }(Q)$ and defining

$$
\delta^{*}=\min \left\{\frac{\min \left\{\lambda_{\min }(Q) / 2, b_{1}, b_{2}\right\}}{b_{1} M_{1}+b_{2} M_{2}+b_{2} M_{3}+2 b_{1}+2 b_{2}}, \frac{1}{M_{3}}, 1\right\}
$$

we obtain the existence of a positive constant $\mu$ such that, provided $|\dot{D}(t)|<\delta^{*}, t \geq 0$,

$$
\forall t \in \mathbb{R}_{+}, \quad \dot{V}(t) \leq-\mu V_{0}(t)
$$

Finally, observing that both

$$
\begin{array}{r}
\min \left\{\lambda_{\min }(P), b_{1} \underline{D}, b_{2} \underline{D}\right\} V_{0}(t)=\eta_{1} V_{0}(t) \leq V(t) \\
V(t) \leq \max \left\{\lambda_{\max }(P), 2 b_{1} \bar{D}, 2 b_{2} \bar{D}\right\} V_{0}(t)=\eta_{2} V_{0}(t) \\
\text { one can deduce that } \forall t \geq 0, \quad V_{0}(t) \leq \frac{\eta_{2}}{\eta_{1}} V_{0}(0) e^{-\frac{\mu}{\eta_{2}} t}
\end{array}
$$

\section{REFERENCES}

Z. Artstein. Linear systems with delayed controls: a reduction. IEEE Transactions on Automatic Control, 27(4):869-879, 1982.

D. Bresch-Pietri and M. Krstic. Adaptive trajectory tracking despite unknown input delay and plant parameters. Automatica, 45(9):2074-2081, 2009.

D. Bresch-Pietri, J. Chauvin, and N. Petit. Adaptive backstepping controller for uncertain systems with unknown input time-delay. application to SI engines. In Proc. of the Conference on Decision and Control, 2010.

D. Bresch-Pietri, J. Chauvin, and N. Petit. Prediction-based feedback control of a class of processes with input-varying delay. In Proc. of the American Control Conference, 2012.

D. Bresch-Pietri, J. Chauvin, and N. Petit. Adaptive control scheme for uncertain time-delay systems. Automatica, To appear.

M. Chèbre, Y. Creff, and N. Petit. Feedback control and optimization for the production of commercial fuels by blending. Journal of Process Control, 20(4):441-451, 2010.

K. Gu and S. I. Niculescu. Survey on recent results in the stability and control of time-delay systems. Journal of Dynamic Systems, Measurement, and Control, 125:158, 2003.

A. Halanay. Differential equations: Stability, oscillations, time lags, volume 23. Academic Press, 1966.
Q. L. Han and K. Gu. Stability of linear systems with timevarying delay: a generalized discretized lyapunov functional approach. Asian Journal of Control, 3(3):170-180, 2001.

J. Harmand and D. Dochain. The optimal design of two interconnected (bio) chemical reactors revisited. Computers \& chemical engineering, 30(1):70-82, 2005.

Y. He, Q. G. Wang, C. Lin, and M. Wu. Delay-range-dependent stability for systems with time-varying delay. Automatica, 43 (2):371-376, 2007.

A. Ivanov, E. Liz, and S. Trofimchuk. Halanay inequality, yorke $3 / 2$ stability criterion, and differential equations with maxima. Tohoku Mathematical Journal, 54(2):277-295, 2002.

M. Jankovic. Recursive predictor design for linear systems with time delay. In American Control Conference, 2008, pages 4904-4909. IEEE, 2008.

X. Jiang and Q. L. Han. New stability criteria for linear systems with interval time-varying delay. Automatica, 44(10):26802685, 2008. ISSN 0005-1098.

M. Krstic. Boundary control of PDEs: A course on backstepping designs. Society for Industrial and Applied Mathematics Philadelphia, PA, USA, 2008a.

M. Krstic. Lyapunov tools for predictor feedbacks for delay systems: Inverse optimality and robustness to delay mismatch. Automatica, 44(11):2930-2935, 2008b.

M. Krstic. Delay compensation for nonlinear, adaptive, and PDE systems. Birkhauser, 2009.

Y. Liu and L.-S. Hu. New stabilization method for linear systems with time-varying input delay. In American Control Conference, 2011.

A. Manitius and A. Olbrot. Finite spectrum assignment problem for systems with delays. Automatic Control, IEEE Transactions on, 24(4):541-552, 2002. ISSN 0018-9286.

S. Mondié and W. Michiels. A safe implementation for finite spectrum assignment: robustness analysis. In Proc. of the 42nd IEEE Conference on Decision and Control (CDC2003), Hawaii, USA, 2003.

Y. S. Moon, P. G. Park, and W. H. Kwon. Robust stabilization of uncertain input-delayed systems using reduction method. Automatica, 37(2):307-312, 2001.

M. T. Nihtila. Finite pole assignment for systems with timevarying input delays. In 30th IEEE Conference on Decision and Control, pages 927-928. IEEE, 1991.

N. Petit, Y. Creff, and P. Rouchon. Motion planning for two classes of nonlinear systems with delays depending on the control. In Proceedings of the 37th IEEE Conference on Decision and Control, volume 1, pages 1007-1011. IEEE, 1998.

J.-P. Richard. Time-delay systems: an overview of some recent advances and open problems. Automatica, 39(10):16671694, 2003.

M. Sbarciog, R. De Keyser, S. Cristea, and C. De Prada. Nonlinear Predictive Control of processes with variable time delay. A temperature control case study. In Proc. of the IEEE International Conference on Control Applications, pages 1001-1006. IEEE, 2008.

O. J. M. Smith. A controller to overcome dead time. ISA Journal, 6(2):28-33, 1959.

D. Yue and Q. L. Han. Delayed feedback control of uncertain systems with time-varying input delay. Automatica, 41(2): 233-240, 2005.

Q. C. Zhong. Robust control of time-delay systems. Springer Verlag, 2006. 